\title{
Ödipus vs. Big-Five
}

Kann eine psychoanalytisch fundierte

Persönlichkeitsdiagnostik einen inkrementellen Beitrag über die Big-Five-Persönlichkeitsfacetten hinaus zur Vorhersage von psychischer Gesundheit und Zufriedenheit am Arbeitsplatz liefern?

\author{
Hannes Gisch ${ }^{1} \oplus$, Johannes Zimmermann² und Thomas Kretschmar ${ }^{1}$ \\ ${ }^{1}$ Mind Institute SE, Berlin \\ ${ }^{2}$ Institut für Psychologie, Universität Kassel
}

Zusammenfassung: Bisherige Studien zeigen, dass individuelle Unterschiede in der psychischen Gesundheit von Mitarbeiter_innen teilweise durch Persönlichkeitsunterschiede erklärt werden können. Einer psychoanalytischen Perspektive folgend, ist die Persönlichkeit durch die spezifische Form der Bewältigung innerer Konflikte geprägt. Die Studie überprüft, ob diese Bewältigungsmodi einen Beitrag zur Erklärung individueller Unterschiede in der psychischen Gesundheit liefern können. Dazu wurden die Zusammenhänge zwischen verschiedenen Bewältigungsmodi und der psychischen Gesundheit von erwerbstätigen Personen, operationalisiert durch Burnout, Arbeitsengagement und Arbeitszufriedenheit sowie die inkrementelle Validität gegenüber den Big-Five-Persönlichkeitsfacetten untersucht. Die Erhebung der Bewältigungsmodi erfolgte mittels des neu entwickelten OPD-Konfliktfragebogens (OPD-KF). Die Zusammenhänge wurden mit Strukturgleichungsmodellen an einer hinsichtlich Alter, Geschlecht und Wohnort merkmalsspezifisch-repräsentativen Stichprobe der deutschen erwerbstätigen Bevölkerung ( $N=545)$ untersucht. Der OPD-KF zeigte überwiegend zufriedenstellende Ergebnisse für interne Konsistenz und Dimensionalität, erwartungsgemäße Zusammenhänge mit den Outcomevariablen sowie inkrementelle Validität. Die Ergebnisse werden diskutiert und Vorschläge zur notwendigen Weiterentwicklung des Instrumentes geliefert.

Schlüsselwörter: Innere Konflikte, Bewältigung, psychische Gesundheit, Burnout, Arbeitsengagement, Arbeitszufriedenheit

Oedipus Versus the Big Five: Do Psychoanalytically Based Assessments of Internal Conflicts Provide Incremental Validity Over the Big Five Personality Facets for the Prediction of Employees' Mental Health and Job Satisfaction?

Abstract: Previous studies show that individual differences in the mental health of employees can be partially explained by differences in personality traits. From a psychoanalytic perspective, personality is shaped by the style that an individual uses to cope with internal conflicts. The relationships between coping modes and mental health of employees (operationalized by burnout, work engagement, and job satisfaction), as well as the incremental validity of coping modes controlling for Big Five personality facets, were examined using structural equation modeling. Coping modes were assessed using the Operationalized Psychodynamic Diagnosis (OPD)-Conflict Questionnaire (OPD$\mathrm{CQ}$ ). The study was carried out on a representative sample of the German working population taking into account age, gender, and place of residence $(N=545)$. The OPD-CQ showed predominantly satisfactory results for internal consistency and dimensionality, expected correlations with the outcome variables, as well as incremental validity. The results are discussed and suggestions for further development of the instrument are provided.

Keywords: internal conflicts, coping, mental health, burnout, work engagement, job satisfaction

Die psychische Gesundheit von Mitarbeiter_innen und ihre Einflussfaktoren stehen zunehmend im Fokus gesellschaftlicher Diskussionen (Bundesministerium für Arbeit und Soziales, 2017) und wissenschaftlicher Forschung (Rau \& Buyken, 2015). Neben der Betrachtung potenzieller Einflüsse auf die psychische Gesundheit auf den Ebenen Team, Führung, Arbeitsplatz und Organisation, las- sen sich Einflüsse in Form von Belastungen und Ressourcen auf der Ebene des Individuums untersuchen (Turgut, Michel \& Sonntag, 2014). In der arbeits- und organisationspsychologischen Forschung werden als Vulnerabilitätsfaktoren für erlebte Belastungen die Persönlichkeitsdimension Neurotizismus oder emotionsbezogene Coping-Stile beschrieben (Langelaan, Bakker, van Doornen 
\& Schaufeli, 2006; Lazarus \& Folkman, 1984). Als individuelle Ressourcen gelten Selbstwirksamkeit, Optimismus oder Kontrollüberzeugungen (Schlett, Pauls \& Soucek, 2018; Spector et al., 2002).

Die vorliegende Untersuchung möchte die Forschungslage um eine psychoanalytische Perspektive erweitern, die den inneren Konflikt ins Zentrum ihrer Persönlichkeitskonzeptes stellt (Thomä \& Kächele, 2006). Mit inneren Konflikten eines Individuums sind hier innerseelische Zusammenstöße gegensätzlicher Motive gemeint, die zu zeitlich überdauernden festgelegten Erlebnis- und Verhaltensmustern führen, was zumeist nicht bewusst ist und kaum willentlich beeinflusst werden kann (Arbeitskreis OPD, 2011). Es wird angenommen, dass diese inneren Konflikte auch im Arbeitskontext wirksam werden und sich auf die psychische Gesundheit von Mitarbeiter_innen auswirken (Benecke \& Möller, 2013).

Im Zuge voranschreitender Operationalisierungs- und Manualisierungsbestrebungen innerhalb der psychoanalytischen Forschungslandschaft (Arbeitskreis OPD, 2011) und der Zurückweisung der These der Unvereinbarkeit von Psychoanalyse und psychometrischer Forschung (Sell \& Warsitz, 2018) werden zunehmend auch quantitativempirische Forschungsmethoden in die Untersuchung zentraler Bestandteile psychoanalytischer Theorie einbezogen.

Die vorliegende Studie hat sich diese Entwicklung zunutze gemacht und anhand des kürzlich entwickelten OPD-Konfliktfragebogens (OPD-KF, Benecke et al., 2018) die individuellen Zusammenhänge verschiedener Bewältigungsmodi innerer Konflikte und der psychischen $\mathrm{Ge}$ sundheit von Mitarbeiter_innen untersucht. Hierzu wurde eine merkmalsspezifisch-repräsentative Stichprobe für Alter, Geschlecht und Wohnort der erwerbstätigen deutschen Bevölkerung erhoben. Unter Einsatz von Strukturgleichungsmodellen wurden die Zusammenhänge zwischen verschiedenen Bewältigungsmodi innerer Konflikte und der psychischen Gesundheit, die durch Burnout, Arbeitsengagement und Arbeitszufriedenheit operationalisiert wurde, untersucht. Außerdem wurde ermittelt, ob der OPD-KF im Vergleich mit den etablierten Big-FivePersönlichkeitsfacetten zusätzliche Varianz hinsichtlich der Vorhersage der untersuchten Outcomes aufklärt.

Das Ziel der Untersuchung bestand darin, mittels eines psychometrischen Vorgehens, die bisherige arbeits- und organisationspsychologische Forschung zu individuellen Einflussfaktoren hinsichtlich der psychischen Gesundheit von Mitarbeiter_innen um eine psychoanalytische Perspektive zu erweitern und so zu einer Annäherung beider Forschungsrichtungen beizutragen.

\section{Der innere Konflikt}

Der innere Konflikt nimmt eine zentrale Rolle in der psychoanalytischen Theorie ein. Die Psychoanalyse betrachtet innere Konflikte als konstitutiv für das menschliche Leben und versteht den Menschen als ein „Konfliktwesen“ (Schüßler, 2014, S. 489). Freud (1894a) entdeckte bei seinen Patient_innen „unverträgliche Vorstellungen“, die miteinander in Konflikt gerieten und mit negativen Affekten einhergingen. Er beobachtete, wie die (zumeist peinlichen) Vorstellungen von seinen Patient_innen durch den Mechanismus der Verdrängung abgewehrt und dadurch vom Bewusstsein ferngehalten wurden, was letztendlich zu Symptombildungen führte. Im Gegensatz zum psychologischen Konfliktbegriff, handelt es sich im psychoanalytischen Verständnis also um vorwiegend unbewusste innere Konflikte (Arbeitskreis OPD, 2011).

Der klassischen psychoanalytischen Auffassung folgend, können Konflikte zwischen psychischen Instanzen (Ich, Es, Über-Ich) oder innerhalb dieser auftreten (Laplanche \& Pontalis, 1989). Weiterhin können innere Konflikte sich zwischen verschiedenen Triebkräften (Lebensund Todestrieb) abspielen. Nachfolgende psychoanalytische Ansätze erweiterten die zugrundeliegenden (Trieb-) Kräfte um basale Motivationssysteme wie z.B. Bindung (Bowlby, 1969), Selbstwert (Kohut, 1973) und Individuation (Mentzos, 1982).

Das Auftreten innerer Konflikte und deren Abwehr werden als ubiquitär und nicht zwingend pathogen angesehen, sind jedoch häufig ursächlich für neurotische Entwicklungen, die sich in Symptombildungen, Verhaltensoder Charakterstörungen äußern können (Laplanche \& Pontalis, 1989). Benecke und Möller (2013) schlagen eine Betrachtung innerer Konflikte auch bei Fragestellungen im wirtschaftlichen Kontext vor (z.B. im Recruiting, im Coaching und in der Führungskräfteentwicklung). Sie stellen heraus, dass innere Konflikte dem Erleben und Verhalten des Menschen generell zugrunde liegen, seine Persönlichkeit bestimmen und daher auch im beruflichen Kontext wirksam werden.

\section{Zur Operationalisierung innerer Konflikte}

Um die inneren Konflikte einer systematisch-wissenschaftlichen Untersuchung auch im wirtschaftlichen Kontext zugänglich zu machen, stellt sich die Frage nach einer Kategorisierung innerer Konflikte. Hierzu ist festzustellen, dass die psychoanalytische Hermeneutik traditionell allgemeine Gesetzmäßigkeiten gegenüber der Erfassung der Singularität und Gesamtheit eines Falles zurückstellen (Sell \& Warsitz, 2018). 
Die Komplexität des psychoanalytischen Konfliktbegriffes führte lange Zeit dazu, dass eine psychometrische Untersuchung und eine dafür notwendige Kategorisierung innerer Konflikte vernachlässigt wurde. Ausgehend von der Forderung nach einer stärkeren Operationalisierung psychoanalytischer Konzepte (Schneider et al., 2018) und einer Integration psychoanalytischer und psychometrischer Forschung (Sell \& Warsitz, 2018), wurde diese Forschungslücke durch die Entwicklung von psychodynamischen Diagnostikmanualen (Arbeitskreis OPD, 2011) und psychometrischer Fragebögen (Benecke et al., 2018; Ehrenthal et al., 2012) geschlossen.

\section{Der OPD-Konfliktfragebogen (OPD-KF)}

Zur Erfassung der Bewältigungsmodi innerer Konflikte wurde in dieser Untersuchung der OPD-Konfliktfragebogen (OPD-KF, Benecke et al., 2018) eingesetzt. Der OPD-KF basiert auf dem Manual „Operationalisierte Psychodynamische Diagnostik“ (OPD-2, Arbeitskreis OPD, 2011), das zur systematischen klinischen Diagnostik und Therapieplanung hinsichtlich zentraler psychoanalytischer Dimensionen eingesetzt werden kann. Der OPD-KF ermöglicht die Erfassung der Bewältigungsmodi von sechs inneren Konflikten (K1-K6) und der Skala „Abgewehrte Gefühls- und Konfliktwahrnehmung“ (KO). Für jeden der Konflikte K1 bis K6 wird jeweils ein aktiver (a) und ein passiver (p) Bewältigungsmodus getrennt voneinander erhoben. Der Bewältigungsmodus umfasst prototypische Versuche des Individuums, den zugrundeliegenden inneren Konflikt zu lösen. Dies geschieht, indem das Individuum sich gewissermaßen auf eine Seite des Konfliktes schlägt und dadurch die andere befürchtete Seite vermeidet. Diese Versuche äußern sich in typischen Überzeugungen über sich und andere, spezifischen Beziehungsmustern und dem Vorherrschen bestimmter Emotionen.

Zur Konstruktvalidität der OPD-Konflikte liegen bisher vor allem Hinweise basierend auf klinischen Ratings durch Fremdbeurteiler_innen für die am häufigsten diagnostizierten Konflikte K1 bis K4 (Kaufhold et al., 2017, 2019) vor. Für diese Konflikte zeigen sich erwartungsgemäße Korrelate mit Persönlichkeits-, Selbstkonzept- und Selbstwertskalen sowie zu Instrumenten, die interpersonale Probleme oder Bindungsstile erfassen (Pieh, Frisch, Meyer, Loew \& Lahmann, 2009; Schneider, Mendler, Heuft \& Burgmer, 2008).

Im Folgenden sollen die inneren Konflikte mit ihren beiden Bewältigungsmodi im Rückgriff auf die Operationalisierungen in der OPD-2 kurz skizziert werden. Eine ausführliche Beschreibung findet sich beim Arbeitskreis
OPD (2011). Der Konflikt zwischen Individuation und Abhängigkeit äußert sich bei aktiver Bewältigung in dem übermäßigen Streben nach Selbstständigkeit und Distanz, um die existenzielle Angst vor Nähe und Vereinnahmung zu vermeiden (Konfliktmodus: K1a). Personen, die denselben Konflikt passiv bewältigen, wehren ihre Angst vor Verlust und Einsamkeit durch das übermäßige Streben nach enger Beziehung und Nähe ab (K1p). Eine aktive Bewältigung des Konfliktes zwischen Unterwerfung und Kontrolle geht mit der Tendenz einher, aus der Angst von anderen bestimmt $\mathrm{zu}$ werden, andere dominieren und Kontrolle über sie ausüben zu wollen (K2a). Individuen, die denselben Konflikt passiv bewältigen, tendieren dagegen zu unterwürfigem und gefügigem Verhalten (K2p). Im Mittelpunkt des Konfliktes zwischen Versorgung und Autarkie stehen übermäßige Wünsche nach Versorgung und Geborgenheit, sowie deren Abwehr. Eine aktive Bewältigung des Konfliktes geht mit einem anspruchslosen Verzicht und der Tendenz, es anderen recht zu machen einher (K3a). Die passive Bewältigung äußert sich in anklammerndem und forderndem Verhalten (K3p). Im Fokus des Selbstwertkonflikts stehen gegensätzliche Bewältigungsversuche zur Stabilisierung des eigenen Selbstwertgefühls. Eine aktive Bewältigung ist durch eine forcierte Selbstsicherheit gegenüber anderen geprägt (K4a). Die passive Bewältigung (K4p) äußert sich in einem Selbstbild, im Vergleich zu anderen weniger wertvoll zu sein. Individuen, die den Schuldkonflikt aktiv bewältigen, wehren Schuldgefühle $\mathrm{ab}$, indem sie sich anklagend verhalten und die Schuld häufig bei anderen suchen (K5a). Eine passive Bewältigung äußert sich in der Neigung zu Selbstvorwürfen und einer übermäßigen Schuldübernahme (K5p). Im Fokus des Ödipalen Konflikts stehen Bedürfnisse nach Aufmerksamkeit, Bewunderung und Anerkennung als Frau oder Mann. Individuen, die den Konflikt aktiv bewältigen, begeben sich häufig in Situationen, die durch Erotik und Konkurrenz geprägt sind (K6a). Eine passive Bewältigung geht dagegen mit einer Vermeidung dieser Situationen einher (K6p).

\section{Der innere Konflikt und psychische Gesundheit am Arbeitsplatz}

In der OPD-2 werden Konfliktmodi generell als dysfunktionale Bewältigungsstile verstanden (Arbeitskreis OPD, 2011). Diese theoretische Grundannahme konnte in der Vorgängerstudie, in der die Bewältigung innerer Konflikte erstmalig mittels eines Selbstbeschreibungsfragebogens untersucht wurde, empirisch größtenteils gestützt werden (Benecke et al., 2018). Genauer gesagt fanden Benecke et al. (2018) für die Konfliktmodi K1a, K1p, K2p, K3a, K3p, $\mathrm{K} 4 \mathrm{p}, \mathrm{K} 5 \mathrm{p}$ und $\mathrm{K} 6 \mathrm{p}$ erwartungsgemäß positive Zusam- 
menhänge mit der allgemeinen psychischen Beschwerdebelastung sowie negative Zusammenhänge (außer K3p) mit der allgemeinen Lebenszufriedenheit. Für die Konfliktmodi K4a und K5a zeigten sich allerdings nicht erwartungsgemäße Zusammenhänge: Beide Konfliktmodi waren mit funktionalen Outcomes, im Sinne einer verminderten Beschwerdebelastung und einer erhöhten Lebenszufriedenheit assoziiert (Benecke et al., 2018). Die Autoren führen diese unerwarteten Ergebnisse u. a. auf charakteristische Unterschiede zwischen Selbst- und Fremdbericht zurück (Benecke et al., 2018).

In der vorliegenden Studie werden diese Vorbefunde zu Zusammenhängen zwischen den Bewältigungsmodi innerer Konflikte und psychischer Gesundheit aufgegriffen und hinsichtlich des spezifischen Kontexts des Arbeitsplatzes konkretisiert. Hierzu werden folgende übergeordnete Fragestellungen verfolgt, die eine Untersuchung der psychometrischen Eigenschaften des OPD-KF einschließen:

1. Erreichen die OPD-KF-Skalen zufriedenstellende Ergebnisse hinsichtlich der internen Konsistenz und der Dimensionalität? (Reliabilität und Dimensionalität)

2. Bestehen erwartungsgemäße Zusammenhänge zwischen den OPD-KF-Skalen und Burnout, Arbeitsengagement und Arbeitszufriedenheit? (Kriteriumsvalidität)

3. Wird durch die Hinzunahme der OPD-KF-Skalen die Varianzaufklärung gegenüber den Big-Five-Persönlichkeitsfacetten bezüglich der Vorhersage von Burnout, Arbeitsengagement und Arbeitszufriedenheit erhöht? (Inkrementelle Validität)

Die aus den Vorbefunden abgeleiteten Annahmen sollen im Folgenden hinsichtlich der Kriteriumsvariablen gesondert dargestellt werden.

\section{Burnout}

Eine einheitliche Definition von Burnout gestaltet sich schwierig (Burisch, 2014; Geuenich, 2016). Als Kernmerkmale werden jedoch emotionale Erschöpfung, Depersonalisation (im Sinne einer gefühlten Distanzierung zu anderen) sowie ein Gefühl von Ineffektivität und verminderter Leistungsfähigkeit beschrieben, die aus einer prolongierten Erfahrung von interpersonalem Stress bei der Arbeit entstehen (Maslach, Schaufeli \& Leiter, 2001). Neben negativen Auswirkungen auf individueller Ebene, wird Burnout im organisationalen Kontext mit erhöhter Fluktuation, Absentismus, verminderten Interaktionen mit Klient_innen sowie verminderter Arbeitsleistung in Verbindung gebracht (Swider \& Zimmerman, 2010).

Die aus individuellen Konfliktlagen entstammende innere Zerrissenheit ist mit der Angespanntheit, die bei einem Burnout auftritt, assoziiert und wird als eine mögliche Ursache diskutiert (Burisch, 2014). Nach Wissen der
Autoren liegen keine empirischen Untersuchungen zu den Zusammenhängen zwischen Bewältigungsmodi innerer Konflikte und Burnout vor. Ausgehend von einer konzeptionellen Nähe zwischen der psychischen Beschwerdebelastung und Burnout, wurden in der vorliegenden Untersuchung analoge Zusammenhänge zu der Studie von Benecke et al. (2018) postuliert:

- Hypothese 1.1: Die Konfliktmodi K1a, K1p, K2p, K3a, $\mathrm{K} 3 \mathrm{p}, \mathrm{K} 4 \mathrm{p}, \mathrm{K} 5 \mathrm{p}$ und $\mathrm{K} 6 \mathrm{p}$ sind positiv korreliert mit Burnout.

- Hypothese 1.2: Die Konfliktmodi K4a und K5a sind negativ korreliert mit Burnout.

\section{Arbeitsengagement}

Arbeitsengagement wird als ein positiver und erfüllender Gefühlszustand beschrieben, der durch Vitalität, Einsatz, bzw. Hingabe und einem Aufgehen in der Arbeit gekennzeichnet ist (Schaufeli, Salanova, González-romá \& Bakker, 2002). Es handelt sich hierbei um einen anhaltenden und durchdringenden affektiv-kognitiven Zustand, der nicht auf ein bestimmtes Objekt, Ereignis, Individuum oder Verhalten beschränkt ist. Arbeitsengagement wird konzeptuell als ein positiver Gegensatz zum Burnout angesehen (Leiter \& Maslach, 2009; Schaufeli et al., 2002). Im Rahmen der stärkeren Fokussierung auf arbeitsbezogene Ressourcen in der Forschung rückte das Konzept des Arbeitsengagements zunehmend in den Vordergrund (Sautier et al., 2015).

Nach Wissen der Autoren liegen keine empirischen Befunde zu den Zusammenhängen zwischen Bewältigungsmodi innerer Konflikte und Arbeitsengagement vor. Ausgehend von der Konzeption als positivem Gegensatz zum Burnout wurden für die Zusammenhänge zwischen den Konfliktmodi und Arbeitsengagement gegenläufig gerichtete Zusammenhänge postuliert:

- Hypothese 2.1: Die Konfliktmodi K1a, K1p, K2p, K3a, K3p, K4p, K5p und K6p sind negativ korreliert mit Arbeitsengagement.

- Hypothese 2.2: Die Konfliktmodi K4a und K5a sind negativ positiv mit Arbeitsengagement.

\section{Arbeitszufriedenheit}

Arbeitszufriedenheit wird als ein angenehmer und positiver Zustand definiert, der aus der Beurteilung der eigenen Arbeit oder den eigenen Arbeitserfahrungen resultiert (Locke, 1976, nach Thoresen, Kaplan, Barsky, Warren \& de Chermont, 2003). Es bestehen positive Zusammenhänge zwischen der allgemeinen Arbeitszufriedenheit und positiven organisationalen Folgen wie Commitment oder Produktivität sowie negative Zusammenhänge mit negativen Konsequenzen wie Kündigungsabsicht oder Fehlzeiten (Turgut et al., 2014). 
Nach Wissen der Autoren liegen keine empirischen Untersuchungen bezüglich der Zusammenhänge zwischen Bewältigungsmodi innerer Konflikte und Arbeitszufriedenheit vor. Aufgrund der konzeptuellen Nähe zwischen Lebens- und Arbeitszufriedenheit, wurde zur Hypothesenbildung auf die Befunde zu den Zusammenhängen zwischen den Konfliktmodi und Lebenszufriedenheit zurückgegriffen (Benecke et al., 2018):

- Hypothese 3.1: Die Konfliktmodi K1a, K1p, K2p, K3a, K4p, K5p und K6p sind negativ korreliert mit Arbeitszufriedenheit.

- Hypothese 3.2: Die Konfliktmodi K4a und K5a sind positiv mit Arbeitszufriedenheit.

\section{Methode}

\section{Studiendesign}

Die Studie wurde als eine querschnittliche Online-Fragebogenuntersuchung über SoSci Survey durchgeführt. Es wurde eine nicht-probabilistische Quotenstichprobe angestrebt, die eine merkmalsspezifische Repräsentativität hinsichtlich Alter, Geschlecht und Wohnort (unabhängige Quotierung) für die deutsche erwerbstätige Bevölkerung gewährleistet. Zur robusten Schätzung der Parameter der Strukturgleichungsmodelle wird ein Stichprobenumfang empfohlen, der zehnmal so groß wie die Anzahl der maximal zu schätzenden Parameter sein sollte (Bentler \& Chou, 1987). Die endgültige Stichprobe $(N=545)$ übererfüllt diese Anforderung für alle berechneten Strukturgleichungsmodelle (min. 17.5 Versuchspersonen pro zu schätzendem Parameter). Nach der Definition der International Labour Organization (ILO) gilt als erwerbstätig, wer mindestens eine Stunde pro Woche gegen Bezahlung gearbeitet hat und mindestens 15 Jahre alt ist (Statistisches Bundesamt, 2020a). Die anvisierten Quoten wurden der Hochrechnung anhand der Bevölkerungsfortschreibung für das Jahr 2016 auf Basis des Zensus 2011 des Statistischen Bundesamtes (2020b) entnommen. Die Rekrutierung der Teilnehmer_innen wurde mithilfe der Crowdsourcing-Plattform clickworker durchgeführt. Für die Teilnahme an der Befragung wurden $5.50 €$ ausgezahlt. Die Durchführung dauerte im Mittel 22.5 Minuten $(S D=7.35)$. Als Einschlusskriterien wurden die informierte Zustimmung zu den Teilnahmebedingungen (angelehnt an Schönbrodt, Gollwitzer \& Abele-Brehm, 2017), das Ausfüllen bis zur letzten Seite des Fragebogens und die Zugehörigkeit zur vorher definierten Altersrange von 20 bis unter 65 Jahren definiert. Da bei der Rekrutierung über Crowdsourcing-Plattformen die Gefahr der nachlässigen Beantwortung der Items besteht (Gosling \& Mason,
2015), wurde als weiteres Einschlusskriterium ein Relative Speed Index $($ RSI $)<2.0$ festgelegt, um übermäßig schnell ausgefüllte Fragebögen auszuschließen (zur Berechnung des RSI siehe Leiner, 2013). Die Datenerhebung erfolgte im August 2018.

\section{Stichprobe}

Die endgültige Stichprobe bestand aus $N=545$ Personen, nachdem $n=44$ Personen wegen eines zu hohen RSI ausgeschlossen wurden. Die vorher festgelegten Quoten bezüglich Alter, Geschlecht und Wohnort konnten annähernd erreicht werden (siehe Tabelle 1). Keiner der Teilnehmer_innen ordnete sich der Kategorie inter/divers zu. Die Altersrange betrug 20 bis 64 Jahre $(M=43.1$, $S D=11.25)$. Die Teilnehmer_innen waren entweder verheiratet oder in einer Lebenspartnerschaft (37\%), ledig, ohne Partner (26.7\%), ledig, mit Partner (24.9\%), geschieden $(7.9 \%)$, getrennt lebend (1.9\%) oder verwitwet (1.7\%). 53.2\% der Teilnehmer_innen schlossen die Schule mit dem Abitur und $18.3 \%$ mit der Fachhochschulreife ab. $0.6 \%$ beendeten die Erweiterte Oberschule (EOS) und $3.5 \%$ die Polytechnische Oberschule. $18.2 \%$ besaßen einen Realschulabschluss, $6.3 \%$ einen Hauptschulabschluss und $0 \%$ keinen schulischen Abschluss. $46.2 \%$ der Teilnehmer_innen hatten einen Hochschulabschluss, $45.9 \%$ entweder Ausbildung, Lehre, oder Fachschule abgeschlossen und $7.9 \%$ besaßen keinen beruflichen Abschluss. $68.5 \%$ der Teilnehmer_innen gaben an, Vollzeit (über 30 Std. pro Woche) zu arbeiten, während sich $22.4 \%$ in Teilzeit (15 bis 30 Std. pro Woche) und $9.1 \%$ in geringfügiger Beschäftigung (unter 15 Std. pro Woche) befanden. Zusätzlich wurde der Sozioökonomische Status (Lampert, Kroll, Müters \& Stolzenberg, 2013) berechnet: Die Teilnehmer_innen mit ausreichenden gültigen Angaben zur Berechnung des SWS $(n=357)$ wurden den Kategorien hoher SES (49.6\%), mittlerer SES (47.9\%) und niedriger SES (2.5\%) zugewiesen.

\section{Messinstrumente}

\section{Der OPD-Konfliktfragebogen (OPD-KF)}

Der OPD-KF umfasst 13 Skalen (K1- 6 mit jeweils aktivem und passivem Bewältigungsmodus sowie K0) mit insgesamt 66 Items. Die Items repräsentieren Aussagen, die für eine aktive bzw. passive Verarbeitung des jeweiligen Konfliktes als typisch angesehen werden. Die Antworten werden mithilfe einer fünfstufigen Skala mit den Kategorien „trifft gar nicht zu“ (0) bis „trifft völlig zu“ (4) erhoben. Bei einigen Skalen (K0, K2a, K2p und K5a) konnten in bisherigen Untersuchungen keine zufriedenstellenden 
Tabelle 1. Beschreibung der Stichprobe

\begin{tabular}{|c|c|c|c|c|c|}
\hline & & Häufigkeit & Stichprobe & Erwerbstätige Deutschland & Differenz \\
\hline \multirow[t]{3}{*}{ Alter $(n=525)$} & 20 bis unter 35 Jahre & 136 & $25.9 \%$ & $28.8 \%$ & $-2.9 \%$ \\
\hline & 35 bis unter 50 Jahre & 200 & $38.1 \%$ & $36.1 \%$ & $2.0 \%$ \\
\hline & 50 bis unter 65 Jahre & 189 & $36.0 \%$ & $35.1 \%$ & $0.9 \%$ \\
\hline \multirow[t]{2}{*}{ Geschlecht $(n=541)$} & weiblich & 262 & $48.4 \%$ & $46.8 \%$ & $1.7 \%$ \\
\hline & männlich & 279 & $51.6 \%$ & $53.2 \%$ & $-1.7 \%$ \\
\hline \multirow[t]{4}{*}{ Region $(n=541)$} & Nord & 72 & $13.3 \%$ & $17.7 \%$ & $-4.4 \%$ \\
\hline & West & 185 & $34.2 \%$ & $34.5 \%$ & $-0.3 \%$ \\
\hline & Ost & 106 & $19.6 \%$ & $17.1 \%$ & $2.5 \%$ \\
\hline & Süd & 178 & $32.9 \%$ & $30.7 \%$ & $2.2 \%$ \\
\hline
\end{tabular}

Anmerkungen: N = 545; Stichprobe: Proz. Anteil gültiger Antworten; Quelle Erwerbstätige in Deutschland: Bevölkerungsfortschreibung des Statistischen Bundesamts für das Jahr 2016 auf Grundlage des Zensus 2011; Einteilung der Regionen: Nord (Bremen, Hamburg, Mecklenburg-Vorpommern, Niedersachsen, Schleswig-Holstein), West (Hessen, Nordrhein-Westfalen, Rheinland-Pfalz, Saarland), Ost (Berlin, Brandenburg, Sachsen, Sachsen-Anhalt, Thüringen) und Süd (Baden-Württemberg, Bayern).

internen Konsistenzen $(\omega<.7)$ erreicht werden (Benecke et al., 2018). Für die Skalen K0, K2a und K2p werden jeweils 1-2 Zusatzitems angegeben, die für die Verbesserung der internen Konsistenz hinzugenommen werden können.

\section{Burnout}

Zur Erfassung der Stress- und der Beschwerdebelastung, die üblicherweise im Rahmen eines Burnout-Syndroms auftreten, wurde die Skala Beruf des ersten Teils der Burnout-Screening-Skalen (BOSS I, Geuenich \& Hagemann, 2014) eingesetzt. Die Skala erfasst mit 10 Items berufliche Beschwerden wie innere Kündigung („,Ich kann mich über Erfolge im Job nicht mehr nachhaltig freuen."), Arbeitsüberforderung (,Ich befinde mich in ständiger Sorge und Anspannung, dass ich meinen Job nicht bewältige.“) oder Verlust der Arbeitsfreude („Die Freude an meiner Arbeit ist mir verloren gegangen."). Die Erhebung erfolgt mittels einer sechsstufigen Antwortskala von „trifft nicht $\mathrm{zu}^{\text {“ }}(0)$, ,trifft kaum zu“ (1), „trifft teilweise zu“ (2), „trifft überwiegend zu“ (3), ,trifft zu“ (4) und „trifft stark zu“ (5). Es werden zufriedenstellende psychometrische Gütekriterien und positive Zusammenhänge zu anderen Stress- und Beschwerdefragebögen berichtet (Geuenich \& Hagemann, 2014). Die interne Konsistenz der Skala Beruf war in der vorliegenden Untersuchung gut $(\omega=.89)$.

\section{Arbeitsengagement}

Zur Erfassung des Arbeitsengagements wurde die deutschsprachige Kurzversion der Utrecht Work Engagement Skala eingesetzt (UWES-9, Sautier et al., 2015). Die Originalversion erfasst die drei Dimensionen Vitalität
(„Wenn ich morgens aufstehe, habe ich Lust zur Arbeit zu gehen.“), Einsatz/Hingabe („Ich bin begeistert von meiner Arbeit.") und Aufgehen in der Arbeit (,Wenn ich arbeite, werde ich völlig mitgerissen.") mit jeweils drei Items. Die drei Dimensionen konnten in der deutschsprachigen Kurzversion nicht repliziert werden, weshalb eine eindimensionale Erfassung des Arbeitsengagements empfohlen wird (Sautier et al., 2015). Die Items werden auf einer siebenstufigen Ratingskala mit den Abstufungen „nie“ (0), „fast nie“ (1), „selten“ (2), „manchmal“ (3), „häufig“ (4), „sehr oft“ (5) und „immer“ (6) bewertet. Es werden gute psychometrische Gütekriterien berichtet (Sautier et al., 2015). In der vorliegenden Studie betrug die interne Konsistenz für die Gesamtskala des UWES-9 $\omega=0.95$.

\section{Arbeitszufriedenheit}

Zur Erfassung der Arbeitszufriedenheit wurde das Item "Wie zufrieden sind Sie gegenwärtig, alles in allem, mit Ihrer momentanen Arbeit?" herangezogen. Das Item ist angelehnt an die „Kurzskala zur Erfassung der Allgemeinen Lebenszufriedenheit (L-1)" (Beierlein, Kovaleva, László, Kemper \& Rammstedt, 2015). Die Skala L-1 ermöglicht eine ausreichend reliable und valide Messung des psychologischen Merkmales der allgemeinen Lebenszufriedenheit (Beierlein et al., 2015). Das Item des L-1 wurde nur im Hinblick auf die Formulierung „mit Ihrem Leben“ für die Zwecke der vorliegenden Untersuchung zu „Wie zufrieden sind Sie gegenwärtig, alles in allem, mit Ihrer momentanen Arbeit?" abgewandelt. Analog zur Skala L-1 wurde eine elfstufige Skala mit den Polen „überhaupt nicht zufrieden“ (0) und „völlig zufrieden“ (10) eingesetzt. 


\section{Persönlichkeit}

Zur Erfassung der Persönlichkeitsmerkmale wurde die deutsche Version des Big Five Inventory-2 eingesetzt (BFI-2, Danner et al., 2019). Der BFI-2 umfasst insgesamt 60 Items. Auf übergeordneter Ebene werden Domänen Extraversion, Verträglichkeit, Gewissenhaftigkeit, Negative Emotionalität (früher: Neurotizismus) und Offenheit abgebildet. Jede der fünf Domänen setzt sich aus drei Facetten zusammen, die jeweils aus vier Items bestehen. Zur Erhebung wird eine fünfstufige Skala mit den Abstufungen "stimme überhaupt nicht zu“ (1), „stimme eher nicht zu“ (2), „teils, teils“ (3), „stimme eher zu“ (4) und „stimme voll und ganz zu" (5) eingesetzt. Für den BFI-2 werden zufriedenstellende Reliabilitäten sowie Hinweise für die Konstrukt- und Kriteriumsvalidität berichtet (Danner et al., 2019). In der vorliegenden Untersuchung waren die Reliabilitäten der einzelnen Facetten überwiegend zufriedenstellend bis gut $(\omega=.73-.89)$. Einzig die Facetten Vertrauen und Verlässlichkeit wiesen nicht zufriedenstellende Reliabilitätswerte auf $(\omega=.65)$.

\section{Statistische Analysen}

Die statistischen Berechnungen wurden mit R (Version 3.5.1 - "Feather Spray", R Core Team, 2018) und den Paketen psych (v. 1.8.4, Revelle, 2018), lavaan (v. 0.6-2, Rosseel, 2012) sowie semTools (v. 0.5-0, Jorgensen, Pornprasertmanit, Schoemann \& Rosseel, 2018) durchgeführt. Zur Bestimmung der internen Konsistenz wurde aufgrund diverser in der Literatur beschriebenen Limitationen auf die Maßzahl Cronbachs a verzichtet (Sijtsma, 2008) und stattdessen McDonalds $\omega$ auf Basis einer eindimensionalen Konfirmatorischen Faktorenanalyse (CFA) herangezogen (Bentler, 1972, 2008). Die McDonalds- $\omega$ Werte wurden analog zu Cronbachs $\alpha$ als zufriedenstellend $(\omega \geq .7)$ oder gut $(\omega \geq .8)$ interpretiert (Dorsch, Wirtz \& Strohmer, 2017). Zur Überprüfung der Dimensionalität der OPD-KF-Skalen wurde eine CFA für ordinale Variablen mit dem Diagonally-Weighted-Least-SquaresSchätzverfahren (DWLS) durchgeführt. Zur Bestimmung der Modellgüte wurden der Comparative Fit Index (CFI) und der Root Mean Square Error of Approximation (RMSEA) herangezogen. Eine akzeptable Modellgüte wurde durch einen CFI-Wert $\geq .95$ und einen RMSEA-Wert $\leq .08$ angezeigt, eine gute Modellgüte durch einen CFI-Wert $\geq .97$ und einen RMSEA-Wert $\leq .05$ (Schermelleh-Engel, Moosbrugger \& Müller, 2003). Aufgrund der in der Literatur beschriebenen Defizite wurde auf die Beurteilung anhand des $\chi^{2}$-Tests verzichtet (SchermellehEngel et al., 2003).

Um bei der statistischen Überprüfung der Zusammenhangshypothesen den Messfehler zu berücksichtigen, wurden Strukturgleichungsmodelle mit dem Full-Information-Maximum-Likelihood-Schätzverfahren (FIML) durchgeführt. Das FIML-Schätzverfahren berücksichtigt alle verfügbaren Datenpunkte und ist herkömmlichen Strategien zum Umgang mit fehlenden Werten wie z.B. dem fallweisen Ausschluss von Personen überlegen (Enders \& Bandalos, 2001). Die Anzahl fehlender Werte war bezüglich aller eingesetzten Messinstrumente auf Itemebene sehr gering $(<1 \%)$. Es wurden jeweils pro Konfliktskala und pro Kriteriumsvariable einzelne Strukturgleichungsmodelle gebildet und berechnet (siehe beispielhafte Darstellung für Konflikt und Burnout in ESM 1: Abbildung E1). Bei den Messinstrumenten BOSS I und UWES-9 wurden jeweils drei gleich große Itemparcel gebildet. Dazu wurden die Items nach den zugrundeliegenden Facetten bzw. Dimensionen gleichmäßig durch eine Zufallsauswahl auf die Itemparcel verteilt (Little, Cunningham, Shahar \& Widaman, 2002). Zur Untersuchung der postulierten Zusammenhänge wurden die standardisierten Parameterschätzungen (bei Standardisierung latenter und manifester Variablen) für die Regressionskoeffizienten der jeweiligen Kriteriums- auf die Prädiktorvariablen herangezogen. Diese lassen sich als PearsonKorrelationen interpretieren und können als kleine $(r \geq .1)$, mittlere $(r \geq .3)$ und große $(r \geq .5)$ Effekte eingeordnet werden (Cohen, 1988).

Zur Überprüfung der inkrementellen Validität der OPD-KF-Skalen gegenüber den Big-Five-Persönlichkeitsfacetten bezüglich der Vorhersage von Burnout, Arbeitsengagement und Arbeitszufriedenheit, wurden zwei multiple Regressionen gerechnet. Im ersten Schritt wurden die 15 Facetten des BFI-2 hinzugefügt. Im zweiten Schritt wurden zusätzlich die 13 Skalen des OPD-KF aufgenommen. Zur Überprüfung, ob die OPD-Konfliktskalen einen inkrementellen Beitrag über die Facetten des BFI-2 hinaus liefern, wurden beide Modelle mithilfe eines $F$-Tests miteinander verglichen. Zur Einordnung der Stärke der inkrementellen Effekte wurde Cohens $f^{2}$ auf Grundlage der adjustierten Determinationskoeffizienten berechnet (kleiner Effekt: $f^{2} \geq .02$, mittlerer Effekt: $f^{2} \geq .15$, großer Effekt: $f^{2} \geq .35$; Cohen, 1988).

\section{Ergebnisse}

\section{Interne Konsistenz und Dimensionalität}

Bezüglich der ersten Fragestellung zu Reliabilität und Dimensionalität des OPD-KF, wurden interne Konsistenz und die Annahme der Eindimensionalität der einzelnen Skalen überprüft. Hinsichtlich der internen Konsistenz erreichten die Skalen K1a, K3p, K4a, K4p, K5p, K6a und 
K6p allesamt zufriedenstellende bis gute $(\omega=.74-.86)$ Ergebnisse (siehe Tabelle 2). Die Skalen K0, K1p, K2a, K2p, K3a und K5a wiesen nicht zufriedenstellende $(\omega<.7)$ Reliabilitätswerte auf. Aufgrund der nicht zufriedenstellenden Werte für die Skalen K0, K2a und K2p wurden für diese die verfügbaren Add-On-Items hinzugefügt und für alle weiteren Berechnungen die erweiterten Skalen verwendet. Dadurch konnten die internen Konsistenzen der drei Skalen erhöht werden, befanden sich jedoch nach wie vor im nicht zufriedenstellenden Bereich.

Bei der Untersuchung der Eindimensionalität mittels CFA erreichten die Skalen K3a, K3p, K4a, K4p, K5a und K5p akzeptable bis gute Modellgütekoeffizienten (siehe ESM 2: Tabelle E1). Bei den Skalen K1a, K1p, K2p, K6a und K6p erreichte jeweils einer der beiden betrachteten Modellgütekoeffizienten den akzeptablen Bereich. Für die Skalen K0 $(\mathrm{CFI}=.749$, RMSEA $=.247)$ und $\mathrm{K} 2 \mathrm{a}(\mathrm{CFI}=.867$, RMSEA $=.155$ ) konnten bezüglich beider Modellgütekoeffizienten keine akzeptablen Ergebnisse erreicht werden.

\section{Zusammenhänge mit Burnout, Arbeits- engagement und Arbeitszufriedenheit}

Bei der Betrachtung der Zusammenhänge zwischen den OPD-Konfliktskalen und den Kriteriumsvariablen (2. Fragestellung) wurden zunächst Maßnahmen zur Verbesserung der Modellgüte vorgenommen. Für die Skalen KO, K1a, K1p, K2a, K3a, K6a und K6p wurden jeweils zusätzliche Residualkorrelationen zwischen den Items zugelassen, deren Formulierungen ähnliche oder gleiche Begriffe beinhalten, was eine gemeinsame Methodenvarianz begünstigt haben könnte. Bei fünf Modellen konnten keine akzeptable Modellgüte erreicht werden (K6a \& Burnout: $\mathrm{CFI}=.918, \mathrm{RMSEA}=.085 ; \mathrm{K} 3 \mathrm{a} \&$ Arbeitsengagement: $\mathrm{CFI}=.940, \mathrm{RMSEA}=.104$; K1p \& Arbeitszufriedenheit: CFI $=.919$, RMSEA $=.088 ;$ K3a \& Arbeitszufriedenheit: $\mathrm{CFI}=.810$, RMSEA $=.120 ;$ K6a \& Arbeitszufriedenheit: $\mathrm{CFI}=.819$, RMSEA $=.122$ ). Bei 11 weiteren Modellen erreichte zumindest einer der betrachteten Modellgütekoeffizienten den akzeptablen Bereich (siehe ESM 2: Tabelle E2). Für die restlichen 23 Modelle ergaben sich akzeptable bis gute Modellgütekoeffizienten. Bei den nicht akzeptablen Modellen sowie den Skalen, bei denen die Annahme der Eindimensionalität verworfen werden musste (KO, K2a), wurde auf eine Interpretation der entsprechenden Zusammenhänge verzichtet.

Hinsichtlich der postulierten Zusammenhänge zeigten sich überwiegend die erwarteten Korrelationen (siehe Tabelle 3, Korrelationen aller eingesetzten Instrumente, siehe ESM 2: Tabelle E3). Große Effekte $(r=.53-.72)$ im Hinblick auf Burnout zeigten sich bei Personen, die sich als abhängig (K1p), unterwürfig (K2p), selbstentwertend
(K4p) oder zur übermäßigen Schuldübernahme neigend (K5p) beschrieben. Bei Personen, die angaben, den Selbstwert- (K4a) und Schuldkonflikt (K5a) aktiv zu bewältigten, zeigten sich die erwarteten negativen Zusammenhänge mit Burnout $(r=-.21--.22)$ sowie die erwarteten positiven Zusammenhänge mit Arbeitsengagement und Arbeitszufriedenheit $(r=.15-.48)$. Nicht erwartungsgemäße Zusammenhänge zeigten sich einzig zwischen dem passiv bewältigten Versorgungskonflikt (K3p) und Burnout $(r=-.03, p=.764)$ sowie Arbeitsengagement $(r=.12, p=0.04)$.

\section{Zusätzliche Varianzaufklärung des OPD-KF gegenüber den Big-Five-Persönlichkeitsfacetten}

Hinsichtlich der dritten Fragestellung (inkrementelle Validität) zeigten sich nach Aufnahme der OPD-KF-Skalen jeweils statistisch signifikante Verbesserungen der Vorhersage von Burnout, Arbeitsengagement und Arbeitszufriedenheit gegenüber den Modellen nur mit den Big-Five-Persönlichkeitsfacetten als Prädiktoren (wegen fragwürdiger Eindimensionalität wurde auf die Hinzunahme der Skalen KO und K2a verzichtet). Der Anteil der aufgeklärten Varianz stieg bei Burnout um $7 \%$ auf $45 \%$ $\left(f^{2}=.13, F[11,518]=7.115, p<.001\right)$, bei Arbeitsengagement um $2.5 \%$ auf $37.7 \%\left(f^{2}=.04, F[11,518]=2.923\right.$, $p<.001)$ und bei Arbeitszufriedenheit um $2.3 \%$ auf $29.8 \%\left(f^{2}=.03, F[11,518]=2.559, p<.01\right)$. Die Zuwächse in den Varianzaufklärungen entsprachen für die Vorhersage von Burnout annähernd einem mittleren Effekt sowie für Arbeitsengagement und Arbeitszufriedenheit jeweils kleinen Effekten (siehe ESM 2: Tabelle E4).

\section{Diskussion}

Das Ziel der vorliegenden Studie bestand darin, die psychometrischen Eigenschaften des kürzlich entwickelten OPD-Konfliktfragebogens (OPD-KF, Benecke et al., 2018) sowie die Zusammenhänge zwischen verschiedenen Bewältigungsmodi innerer Konflikte und den arbeits- und organisationspsychologischen Kriteriumsvariablen Burnout, Arbeitsengagement und Arbeitszufriedenheit an einer merkmalsspezifisch-repräsentativen Stichprobe der deutschen erwerbstätigen Bevölkerung zu untersuchen. Die Studie erweitert die Forschungslage hinsichtlich mehrerer Punkte. Erstens wurde der OPD-KF auf Grundlage von Ad-hoc-Stichproben der Allgemeinbevölkerung bzw. im klinischen Setting entwickelt, d.h. eine Überprüfung der Gütekriterien in einer unabhängigen Stichprobe 


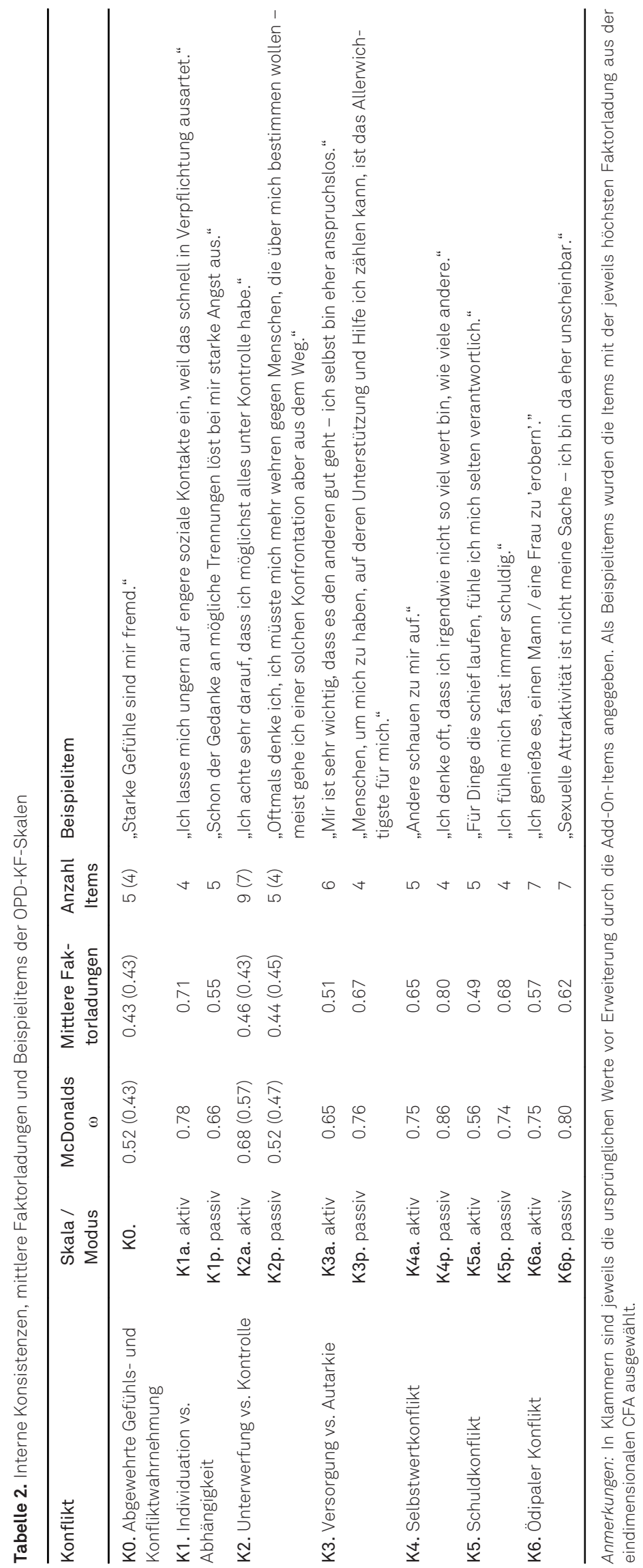


Tabelle 3. Korrelationen der latent modellierten Skalen des OPD-Konfliktfragebogens (OPD-KF) mit Burnout (BOSS I: Beruf), Arbeitsengagement (UWES-9) und Arbeitszufriedenheit

\begin{tabular}{|c|c|c|c|}
\hline & Burnout & Arbeitsengagement & Arbeitszufriedenheit \\
\hline KO & $-0.33 * \star \star$ & $0.37 * * *$ & $0.36^{* * *}$ \\
\hline K1a & $0.42 * \star \star$ & $-0.25 * \star \star$ & $-0.28 * \star \star$ \\
\hline K1p & $0.53^{* * *}$ & $-0.18 * \star \star$ & $-0.20 * * *$ \\
\hline $\mathrm{K} 2 \mathrm{a}$ & -0.02 & $0.49 * * *$ & $0.35^{\star * *}$ \\
\hline$K 2 p$ & $0.72^{\star * \star}$ & $-0.38 * * \star$ & $-0.43 * \star \star$ \\
\hline K3a & $0.19 * \star$ & 0.00 & $-0.15^{\star}$ \\
\hline K3p & -0.03 & $0.12^{\star}$ & $0.12^{\star}$ \\
\hline K4a & $-0.22 * \star \star *$ & $0.48 * * *$ & $0.44^{\star \star *}$ \\
\hline K4p & $0.62^{* * \star}$ & $-0.39 * * \star$ & $-0.44 * \star \star$ \\
\hline $\mathrm{k} 5 \mathrm{a}$ & $-0.21 * *$ & $0.15^{\star}$ & $0.20 * \star$ \\
\hline$K 5 p$ & $0.56^{* * *}$ & $-0.18 * *$ & $-0.27 * \star \star$ \\
\hline K6a & 0.10 & $0.16^{* \star}$ & $0.16^{* *}$ \\
\hline K6p & $0.30 * * *$ & $-0.27 * \star \star$ & $-0.26 * \star \star$ \\
\hline
\end{tabular}

Anmerkungen: Die Korrelationen basieren auf den standardisierten Parameterschätzungen für den Regressionskoeffizienten (bei Standardisierung latenter und manifester Variablen), die kursiv dargestellten Werte kennzeichnen eine nicht akzeptable Modellgüte hinsichtlich der Überprüfung der Eindimensionalität der Skala oder der Strukturgleichungsmodelle zur Berechnung der Zusammenhänge, ${ }^{*} p<.05,{ }^{* *} p<.01,{ }^{* * *} p<.001$.

steht noch aus (Benecke et al., 2018). Zweitens fehlen bisher Untersuchungen zur inkrementellen Validität gegenüber wissenschaftlich etablierten Persönlichkeitsdimensionen (Benecke et al., 2018). Drittens liegen bisher keine arbeits- und organisationspsychologischen Untersuchungen hinsichtlich der Zusammenhänge zwischen verschiedenen Konfliktmodi und Burnout, Arbeitsengagement und Arbeitszufriedenheit vor.

Unsere Ergebnisse zeigen, dass der OPD-KF zumindest für die Mehrheit der Skalen zufriedenstellende Reliabilitätswerte liefert, erwartungsgemäße Zusammenhänge der OPD-KF-Skalen mit Burnout, Arbeitszufriedenheit und Arbeitsengagement bestehen sowie ein Zuwachs an Varianzaufklärung durch die Hinzunahme des OPD-KF gegenüber den Big-Five-Persönlichkeitsfacetten zur Prognose der untersuchten Outcomes erreicht werden kann. Die Studie legt jedoch nahe, dass aufgrund nicht zufriedenstellender Reliabilitätswerte und fragwürdiger Eindimensionalität mehrerer Skalen des OPD-KF eine Weiterentwicklung des Instruments erforderlich ist.

\section{Interne Konsistenz und Dimensionalität}

Bei der Betrachtung der internen Konsistenzen der OPD-KF-Skalen zeigen sich tendenziell geringere Werte als in der Studie von Benecke et al. (2018). Dies könnte darauf zurückzuführen sein, dass die Skalen in der Studie von Benecke et al. (2018) an derselben Stichprobe erstellt und überprüft wurden, was eine Überschätzung der Reliabilitätswerte begünstigt haben könnte. Bei sechs der 13 Skalen erreichten die Werte für die interne Konsistenz jedoch nicht den zufriedenstellenden Bereich. Als Erklärung ließe sich anführen, dass die Skalen jeweils verschiedene Facetten eines zugrundeliegenden Konstruktes abdecken, was wiederrum zu einer Reduzierung der internen Konsistenz führen würde. Hierzu wurde zusätzlich die Dimensionalität der einzelnen Skalen überprüft. Die Ergebnisse legen nahe, dass durch die Erweiterung um die Add-On-Items bei den Skalen KO und K2a die Annahme der Eindimensionalität verworfen werden muss. Beispielsweise beschreiben die Items für K2a einerseits ein starkes Kontrollbedürfnis, andererseits die Motivation Macht und Dominanz auszuüben. Bei fünf weiteren Skalen (K1a, K1p, K2p, K6a und K6p) erscheint die Eindimensionalität aufgrund der Ergebnisse zumindest fragwürdig zu sein. Hinsichtlich der Einordnung der Werte für die interne Konsistenz lässt sich anführen, dass es Hinweise darauf gibt, dass Erhebungen mittels CrowdsourcingPlattformen im Vergleich zu klassischen Erhebungsmethoden niedrigere Reliabilitätswerte liefern (Rouse, 2015).

\section{Zusammenhänge mit Burnout, Arbeits- engagement und Arbeitszufriedenheit}

Zur Überprüfung der Kriteriumsvalidität wurden die $\mathrm{Zu}-$ sammenhänge zwischen den OPD-KF-Skalen und Burnout (BOSS I: Beruf), Arbeitsengagement (UWES-9) und Arbeitszufriedenheit untersucht. Aufgrund der bei einigen Skalen des OPD-KF bereits beschriebenen Einschränkungen hinsichtlich der Eindimensionalität (KO, K2a) sowie der nicht akzeptablen Modellgütekoeffizienten zur Berechnung der Strukturgleichungsmodelle (K1p mit Arbeitszufriedenheit, K3a mit Arbeitsengagement und Arbeitszufriedenheit, K6a mit Burnout und Arbeitszufriedenheit) wurde auf die Interpretation dieser Zusammenhänge verzichtet. Als Erklärung für die mangelhafte Modellpassung ließen sich korrelierte Residuen zwischen einzelnen Items des OPD-KF und den Kriteriumsvariablen anführen.

Die Studie konnte überwiegend die empirischen Befunde aus der Studie von Benecke et al. (2018) replizieren, in der gleich gerichtete Zusammenhänge der OPD-KFSkalen mit konzeptuell nahen Kriteriumsvariablen (psychische Beschwerdebelastung und Lebenszufriedenheit) gezeigt werden konnten. Lediglich der erwartete positive Zusammenhang zwischen der passiven Bewältigung des Versorgungs-Autarkie-Konfliktes (K3p) und Burnout sowie der negative Zusammenhang mit Arbeitsengagement konnten nicht repliziert werden. Dies könnte darauf zurückzuführen sein, dass eine passive Bewältigung des 
Versorgungs-Autarkie-Konfliktes nur in Kombination mit weiteren bisher nicht kontrollierten Faktoren, wie einer geringen sozialen Kompetenz, eines fehlenden sozialen Netzwerks oder dem Vorliegen einer psychischen Störung, mit dysfunktionalen Auswirkungen einhergeht. Dadurch ließe sich erklären, weshalb der Bewältigungsmodus K3p in einer klinischen Stichprobe tendenziell mit dysfunktionalen (Benecke et al., 2018) und in der vorliegenden Studie unter Erwerbstätigen eher mit funktionalen Merkmalen assoziiert ist.

Bei der übergreifenden Betrachtung der statistisch bedeutsamen Zusammenhänge zeigte sich, dass vor allem die passive Bewältigung aller untersuchten Konflikte (mit Ausnahme K3p) sowie die aktive Bewältigung des Individuation-Abhängigkeits-Konfliktes (K1a) mit einer stärkeren Burnout-Belastung sowie vermindertem Arbeitsengagement und Arbeitszufriedenheit einhergingen. Entgegengesetzte Zusammenhänge, i. S. einer verminderten Burnout-Belastung sowie erhöhtem Arbeitsengagement und Arbeitszufriedenheit, zeigten sich für die jeweils aktive Bewältigung des Selbstwert- (K4a) und des Schuldkonfliktes (K5a). Diese Ergebnisse stehen im Einklang mit den gebildeten Hypothesen und replizieren die empirischen Befunde aus vorangegangenen Studien zum OPD-KF (Benecke et al., 2018).

Ausgehend von der OPD-2 wurde bei der Konzeption des OPD-KF ursprünglich davon ausgegangen, dass erhöhte Ausprägungen sowohl bei aktiver als auch bei passiver Bewältigung generell mit einer erhöhten Beschwerdebelastung sowie verminderter Lebenszufriedenheit einhergehen (Arbeitskreis OPD, 2011; Benecke et al., 2018). Die entgegengesetzten empirischen Befunde hinsichtlich der aktiven Bewältigung des Selbstwert- (K4a) und Schuldkonflikts (K5a) werfen die Frage auf, inwieweit hohe Ausprägungen bei diesen beiden Skalen tatsächlich als individuelle Ressourcen anzusehen sind (da sie mit geringerer Belastung sowie erhöhtem Arbeitsengagement und erhöhter Arbeitszufriedenheit einhergingen). Dagegen ließe sich aus psychoanalytischer Perspektive bei Personen, die angeben, von sich überzeugt zu sein (K4a) und dazu tendieren, anderen die Schuld zu geben (K5a) jeweils ein zugrundeliegender innerer Konflikt um Selbstwert und Schuldübernahme annehmen, der mit einer Abwehr von eigenen Schwächen einhergeht (Arbeitskreis OPD, 2011; Kernberg \& Hartmann, 2010). Benecke et al. (2018) führen analog eine inhaltliche Nähe zu narzisstischen (K4a) sowie antisozialen (K5a) Persönlichkeitsstilen an, die mit einem verminderten Problembewusstsein hinsichtlich eigener Persönlichkeitsmerkmale assoziiert sind.

\section{Zusätzliche Varianzaufklärung des OPD-KF gegenüber den Big-Five-Persönlichkeitsfacetten}

Bei der Untersuchung der inkrementellen Validität gegenüber den Big-Five-Persönlichkeitsfacetten zeigten sich jeweils statistisch signifikante Zuwächse in der Varianzaufklärung hinsichtlich der Vorhersage von Burnout, Arbeitsengagement und Arbeitszufriedenheit. Diese Befunde sprechen dafür, dass der OPD-KF eine eigenständige Prognosekraft hinsichtlich der untersuchten arbeits- und organisationspsychologischen Kriteriumsvariablen besitzt und über die BigFive-Persönlichkeitsfacetten hinausgehende Erlebens- und Verhaltensweisen erfasst, wobei herauszustellen ist, dass die Effekte insgesamt eher klein ausfielen.

\section{Stärken, Limitationen und weitere Forschung}

Die vorliegende Studie besitzt diverse Stärken hinsichtlich der Konzeption, der Stichprobe und der eingesetzten statistischen Analysen. Eine Stärke der Studie stellt die Verbindung von psychoanalytischen Konzepten und psychometrischer Forschung dar. Sie folgt damit der Forderung einer zunehmenden Integration beider Forschungsansätze (Sell \& Warsitz, 2018). Ein weiteres Qualitätsmerkmal der Studie stellt die Stichprobe dar, die zum einen merkmalsspezifisch-repräsentativ hinsichtlich Alter, Geschlecht und Wohnort für die deutsche erwerbstätige Bevölkerung war und zum anderen einen zur Überprüfung der voraussetzungsreichen Strukturgleichungsmodelle ausreichend großen Umfang besaß. Die Erhebung mittels Crowdsourcing-Plattformen liefert gegenüber herkömmlichem Vorgehen eine ausreichende Datenqualität, demographisch diversere Teilnehmer_innen und wird zunehmend als eine adäquate Möglichkeit zur Stichprobengenerierung angesehen (Buhrmester, Kwang \& Gosling, 2011; Casler, Bickel \& Hackett, 2013; Lutz, 2016). Bezüglich der eingesetzten statistischen Analysen lässt sich als Stärke anführen, dass durch den Einsatz von Strukturgleichungsmodellen der Messfehler modelliert werden konnte, was einen Vorteil hinsichtlich der Aussagekraft der berichteten Zusammenhänge gegenüber der Berechnung beobachteter (unkorrigierter) Korrelationen darstellt. Außerdem wurde zur Überprüfung der internen Konsistenz der statistisch angemessenere Koeffizient McDonalds $\omega$ herangezogen und auf die Berechnung von Cronbachs a verzichtet.

Als Schwächen der vorliegenden Studie müssen verschiedene Punkte anerkannt werden. Die Erhebung der Prädiktor- und Kriteriumsvariablen erfolgte ausschließlich mittels Selbstbeschreibungsfragebögen, wodurch ein 
erhöhtes Risiko für methodische Verzerrungen im Sinne der common-method-variance besteht (Podsakoff, MacKenzie, Lee \& Podsakoff, 2003). Die Daten lagen nur im Querschnitt vor, was zu entsprechenden Einschränkungen hinsichtlich der Interpretation der Zusammenhangshypothesen führt. Die Stichprobe ist trotz ihrer Größe nicht optimal: Sie besteht im Vergleich zur Zielpopulation der erwerbstätigen deutschen Bevölkerung aus einem größeren Anteil an Personen mit höherer Schulbildung und akademischen $\mathrm{Ab}$ schlüssen. Zusätzlich ist von einer leichten Verzerrung hin zu ledigen gegenüber verheirateten Personen sowie einem erhöhten Anteil an Personen mit einem hohen sozioökonomischen Status auszugehen. Als Nachteile, die bei Erhebungen über Crowdsourcing-Plattform auftreten können, werden in der Literatur geringere Reliabilitätswerte (Rouse, 2015) oder die nachlässige Beantwortung der Items (Gosling \& Mason, 2015) diskutiert.

Eine Weiterentwicklung des OPD-KF erachten wir für zwingend notwendig. Die Ergebnisse der Studie sollten dafür genutzt werden, den Fragebogen hinsichtlich interner Konsistenz und Dimensionalität zu verbessern, indem beispielsweise die problematischen Skalen in Subskalen differenziert werden, die unterschiedliche Facetten des übergeordneten Konstruktes abdecken. Bisher war der OPD-KF mit eindimensionalen Skalen konzipiert worden, um eine hohe inhaltliche Nähe zur OPD zu gewährleisten (Benecke et al., 2018). Zur Frage, ob die beiden Skalen K4a und K5a Ressourcen messen sind weitere Untersuchungen notwendig, die neben Selbstbeschreibungsinstrumenten auch Fremdbeurteilungen einschließen, um Abweichungen zwischen beiden Quellen zu explorieren. Da hierfür nach der OPD-2 geschulte Rater notwendig wären, könnte eine zum OPD-KF analoge Entwicklung eines psychometrischen Instrumentes sinnvoll sein, um eine ökonomischere Erfassung der Fremdperspektive mithilfe verhaltens- und erlebensnaher Items zu ermöglichen. Die OPD-2 bietet dazu bereits ausführliche Informationen hinsichtlich spezieller Verhaltensweisen der Patient_innen sowie Gegenübertragungen (Erleben der Therapeut_innen) für die einzelnen Konfliktmodi, aus denen sich Items für ein Fremdbeurteilungsinstrument ableiten lassen (Kretschmar \& Hamburger, 2019).

In zukünftigen Untersuchungen ist zudem eine Überprüfung der Konstruktvalidität des OPD-KF erforderlich. Es sollten inhaltlich naheliegende Konzepte mit den einzelnen Konfliktskalen in Verbindung gesetzt werden. Denkbar wäre es beispielsweise, die Korrelate mit folgenden Konzepten zu untersuchen: Alexithymie (KO, Bagby, Parker \& Taylor, 1994), Selbstwert (K4, Rosenberg, 1965), Schuld und Scham (K4 bis K6, Tangney, 1990) sowie Stress- und Coping (KO bis K6, Lazarus \& Folkman, 1984). Besonderes Interesse sollte aufgrund der großen konzeptuellen Nähe darauf gelegt werden, die
Theorie der inneren Konflikte mit der Forschung zur Diagnostik impliziter und expliziter Motive sowie zu den Konflikten zwischen beiden Ebenen (Motivdiskrepanzen, siehe Brunstein, Schultheiss \& Grässman, 1998; Kehr, 2004; McClelland, Koestner \& Weinberger, 1989) in Verbindung zu setzen und mögliche Zusammenhänge zu untersuchen. Es gilt außerdem zu beachten, dass die Persönlichkeit aus psychoanalytischer Perspektive nicht nur in der Bewältigung innerer Konflikte, sondern auch in strukturellen Fähigkeiten oder habituellen Beziehungsmustern zum Ausdruck kommt (Arbeitskreis OPD, 2011). Die Konzepte Struktur und Beziehung wurden in dieser Studie ausgeklammert, sollten aber in zukünftigen Untersuchungen miteinbezogen werden (Ehrenthal et al., 2012; Zimmermann, Stasch, Grande, Schauenburg \& Cierpka, 2014).

Zur Frage, ob die inneren Konflikte tatsächlich zeitlich überdauernde Erlebnis- und Verhaltensmuster messen und ob die inneren Konflikte Veränderungen in den Kriteriumsvariablen vorausgehen, könnten längsschnittliche Untersuchungen wertvolle Hinweise liefern. Zusätzlich wären experimentelle Untersuchungen denkbar, um die Frage zu klären, ob durch den OPD-KF tatsächlich unbewusst wirksame Konfliktbewältigungsmodi aus den Antworten der Proband_innen erschlossen werden können oder ob es sich eher um bewusste Persönlichkeitsstile handelt (Benecke et al., 2018).

\section{Elektronische Supplemente (ESM)}

Die elektronischen Supplemente sind mit der OnlineVersion dieses Artikels verfügbar unter https://doi.org/ 10.1026/0932-4089/a000319

ESM 1. Abbildung E1: Beispielhafte Darstellung der Strukturgleichungsmodelle.

ESM 2. Tabellen E1 - E4: Die Tabellen zeigen die Modellgütekoeffizienten zu CFA und Strukturgleichungsmodellen, die bivariaten Korrelationen aller in der Untersuchung eingesetzten Variablen sowie die Ergebnisse der multiplen Regressionsanalysen.

\section{Literatur}

Arbeitskreis OPD. (2011). Operationalisierte Psychodynamische Diagnostik OPD-2. Das Manual für Diagnostik und Therapieplanung (2., überarb. Aufl.). Bern: Hans Huber.

Bagby, R. M., Parker, J. D. A. \& Taylor, G. J. (1994). The twenty-item Toronto Alexithymia Scale: I Item selection and cross-validation of the factor structure. Journal of Psychosomatic Research, 38 (1), 23 - 32. https://doi.org/10.1016/0022-3999(94)90005-1 
Beierlein, C., Kovaleva, A., László, Z., Kemper, C. J. \& Rammstedt, B. (2015). Kurzskala zur Erfassung der Allgemeinen Lebenszufriedenheit (L-1). Zusammenstellung sozialwissenschaftlicher Items und Skalen. https://doi.org/10.6102/zis229

Benecke, C., Henkel, M., Doering, S., Jakobsen, T., Stasch, M., Dahlbender, R. W. et al. (2018). Der OPD-Konfliktfragebogen. Zeitschrift für Psychosomatische Medizin und Psychotherapie, 64, 380 - 393. https://doi.org/10.13109/zptm.2018.64.4.380

Benecke, C. \& Möller, H. (2013). OPD-basierte Diagnostik im Coaching. In H. Möller \& S. Kotte (Hrsg.), Diagnostik im Coaching (S. 183 -198). Berlin: Springer.

Bentler, P. M. (1972). A lower-bound method for the dimensionfree measurement of internal consistency. Social Science Research, 1, 343-357. https://doi.org/10.1016/0049-089X(72) 90082-8

Bentler, P. M. (2008). Alpha, dimension-free, and model-based internal consistency reliability. Psychometrika, 74, 137-143. https://doi.org/10.1007/s11336-008-9100-1

Bentler, P. M. \& Chou, C.-P. (1987). Practical issues in structural modeling. Sociological Methods \& Research, 16 (1), 78-117. https://doi.org/10.1177/0049124187016001004

Bowlby, J. (1969). Attachment and loss. Volume 1: Attachment (2nd ed.). New York, NY: Basic Books.

Brunstein, J. C., Schultheiss, O. C. \& Grässman, R. (1998). Personal goals and emotional well-being: The moderating role of motive dispositions. Journal of Personality and Social Psychology, 75, 494-508. https://doi.org/10.1037/0022-3514.75.2.494

Buhrmester, M., Kwang, T. \& Gosling, S. D. (2011). Amazon's Mechanical Turk: A new source of inexpensive, yet high-quality, data? Perspectives on Psychological Science, 6 (1), 3 - 5. https:// doi.org/10.1177/1745691610393980

Bundesministerium für Arbeit und Soziales. (2017). Weißbuch Arbeiten 4.0. Zugriff am 05.02. 2019 unter https://www.bmas.de/ DE/Service/Medien/Publikationen/a883-weissbuch.html

Burisch, M. (2014). Das Burnout-Syndrom (5. Aufl.). Berlin: Springer. https://doi.org/10.1007/978-3-642-36255-2

Casler, K., Bickel, L. \& Hackett, E. (2013). Separate but equal? A comparison of participants and data gathered via Amazon's MTurk, social media, and face-to-face behavioral testing. Computers in Human Behavior, 29, 2156 - 2160. https://doi.org/ 10.1016/j.chb.2013.05.009

Cohen, J. (1988). Statistical Power Analysis for the Behavioral Sciences (2nd ed.). Hillsdale, NJ: Erlbaum. https://doi.org/ 10.4324/9780203771587

Danner, D., Rammstedt, B., Bluemke, M., Lechner, C., Berres, S., Knopf, T. et al. (2019). Das Big Five Inventar 2. Diagnostica, 65, 121 -132. https://doi.org/10.1026/0012-1924/a000218

Dorsch, F., Wirtz, M. A. \& Strohmer, J. (2017). Lexikon der Psychologie (18., überarb. Aufl.). Bern: Hogrefe.

Ehrenthal, J. C., Dinger, U., Horsch, L., Komo-Lang, M., Klinkerfuß, M., Grande, T. et al. (2012). Der OPD-Strukturfragebogen (OPDSF): Erste Ergebnisse zu Reliabilität und Validität. Psychotherapie Psychosomatik Medizinische Psychologie, 62 (01), 25 - 32. https://doi.org/10.1055/s-0031-1295481

Enders, C. K. \& Bandalos, D. L. (2001). The relative performance of full information maximum likelihood estimation for missing data in structural equation models. Structural Equation Modeling: A Multidisciplinary Journal, 8, 430 -457. https://doi.org/ 10.1207/S15328007SEM0803_5

Freud, S. (1894a). Die Abwehr-Neuropsychosen. Versuch einer psychologischen Theorie der acquirierten Hysterie, vieler Phobien und Zwangsvorstellungen und gewisser hallucinatorischer Psychosen. Neurol. Zbl., 13, GW I, S. $59-74$.

Geuenich, K. (2016). Zusatzdiagnose Burnout. Zugriff am 27.04. 2018 unter https://www.testzentrale.de/themen/klinik/artikel/ Zusatzdiagnose\%20Burnout-311
Geuenich, K. \& Hagemann, W. (2014). Burnout-Screening-Skalen (2., überarb. und erw. Aufl.). Göttingen: Hogrefe.

Gosling, S. D. \& Mason, W. (2015). Internet research in psychology. Annual Review of Psychology, 66, 877 -902. https://doi.org/10. 1146/annurev-psych-010814-015321

Jorgensen, T. D., Pornprasertmanit, S., Schoemann, A. M. \& Rosseel, Y. (2018). semTools: Useful tools for structural equation modeling. Verfügbar unter: https://CRAN.R-project.org/packa ge=semTools

Kaufhold, J., Bahrke, U., Kallenbach, L., Negele, A., Ernst, M., Keller, W. et al. (2019). Wie können nachhaltige Veränderungen in Langzeittherapien untersucht werden? Psyche, 73 (02), $106-$ 133. https://doi.org/10.21706/ps-73-2-106

Kaufhold, J., Negele, A., Leuzinger-Bohleber, M., Kallenbach, L., Ernst, M. \& Bahrke, U. (2017). Zur Konfliktdynamik bei chronischer Depression-Ergebnisse zur Konflikt-und Strukturachse der OPD in der LAC-Studie. Zeitschrift für Psychosomatische Medizin und Psychotherapie, 63, 151 -162. https://doi.org/10. 13109/zptm.2017.63.2.151

Kehr, H. M. (2004). Integrating Implicit motives, explicit motives, and perceived abilities: The compensatory model of work motivation and volition. Academy of Management Review, 29, $479-$ 499. https://doi.org/10.5465/amr.2004.13670963

Kernberg, O. F. \& Hartmann, H.-P. (Hrsg.). (2010). Narzissmus. Grundlagen - Störungsbilder - Therapie (1. Aufl.). Stuttgart: Schattauer.

Kohut, H. (1973). Narzißmus: Eine Theorie der psychoanalytischen Behandlung narzißtischer Persönlichkeitsstörungen (1. Aufl.). Frankfurt am Main: Suhrkamp.

Kretschmar, T. \& Hamburger, A. (2019). Coaching und Supervision: Psychodynamische Beratung von Führungskräften (1. Aufl.). Stuttgart: Kohlhammer.

Lampert, T., Kroll, L., Müters, S. \& Stolzenberg, H. (2013). Messung des sozioökonomischen Status in der Studie zur Gesundheit Erwachsener in Deutschland (DEGS1). Bundesgesundheitsblatt - Gesundheitsforschung - Gesundheitsschutz, 56, 631-636. https://doi.org/10.1007/s00103-012-1663-4

Langelaan, S., Bakker, A. B., Doornen, L. J. P. van \& Schaufeli, W. B. (2006). Burnout and work engagement: Do individual differences make a difference? Personality and Individual Differences, 40, 521 - 532. https://doi.org/10.1016/j.paid.2005.07.009

Laplanche, J. \& Pontalis, J.-B. (1989). Das Vokabular der Psychoanalyse (9. Aufl.). Frankfurt am Main: Suhrkamp.

Lazarus, R. S. \& Folkman, S. (1984). Stress, appraisal, and coping. New York, NY: Springer.

Leiner, D. J. (2013). Too fast, too straight, too weird: post hoc identification of meaningless data in internet. München: Ludwig-Maximilians-Universität.

Leiter, M. P. \& Maslach, C. (2009). Nurse turnover: The mediating role of burnout. Journal of Nursing Management, 17 (3). https:// doi.org/10.1111/j.1365-2834.2009.01004.x

Little, T. D., Cunningham, W. A., Shahar, G. \& Widaman, K. F. (2002). To parcel or not to parcel: Exploring the question, weighing the merits. Structural Equation Modeling: A Multidisciplinary Journal, 9, 151 -173. https://doi.org/10.1207/S15328007 SEM0902_1

Lutz, J. (2016). The validity of crowdsourcing data in studying anger and aggressive behavior: A comparison of online and laboratory data. Social Psychology, 47 (1), 38 -51. https://doi.org/ 10.1027/1864-9335/a000256

Maslach, C., Schaufeli, W. B. \& Leiter, M. P. (2001). Job burnout. Annual Review of Psychology, 52, 397-422. https://doi.org/ 10.1146/annurev.psych.52.1.397

McClelland, D. C., Koestner, R. \& Weinberger, J. (1989). How do self-attributed and implicit motives differ? Psychological Re- 
view, 96, 690-702. https://doi.org/10.1037/0033-295X.96.4. 690

Mentzos, S. (1982). Neurotische Konfliktverarbeitung: Einführung in die psychoanalytische Neurosenlehre unter Berücksichtigung neuer Perspektiven. München: Kindler.

Pieh, C., Frisch, M., Meyer, N., Loew, T. \& Lahmann, C. (2009). Validierung der Achse III (Konflikt) der Operationalisierten Psychodynamischen Diagnostik (OPD). Zeitschrift für Psychosomatische Medizin und Psychotherapie, 55, 263-281.

Podsakoff, P. M., Mackenzie, S. B., Lee, J. Y. \& Podsakoff, N. P. (2003). Common method biases in behavioral research: A critical review of the literature and recommended remedies. Journal of Applied Psychology, 88, 879-903. https://doi.org/10. 1037/0021-9010.88.5.879

R Core Team. (2018). R: A language and environment for statistical computing. Wien: R Foundation for Statistical Computing. Verfügbar unter: https://www.R-project.org/

Rau, R. \& Buyken, D. (2015). Der aktuelle Kenntnisstand über Erkrankungsrisiken durch psychische Arbeitsbelastungen. Zeitschrift für Arbeits- und Organisationspsychologie, 59, 113-129. https://doi.org/10.1026/0932-4089/a000186

Revelle, W. (2018). psych: Procedures for personality and psychological research. Evanston, IL: Northwestern University. Verfügbar unter: https://CRAN.R-project.org/package=psych.

Rosenberg, M. (1965). Society and the adolescent self-image. Princeton, NJ: Princeton University Press.

Rosseel, Y. (2012). lavaan: An R package for structural equation modeling. Journal of Statistical Software, 48 (2), 1 -36. https:// doi.org/10.18637/jss.v048.i02

Rouse, S. V. (2015). A reliability analysis of Mechanical Turk data. Computers in Human Behavior, 43, 304-307. https://doi.org/ 10.1016/j.chb.2014.11.004

Sautier, L. P., Scherwath, A., Weis, J., Sarkar, S., Bosbach, M., Schendel, M. et al. (2015). Erfassung von Arbeitsengagement bei Patienten mit hämatologischen Malignomen: Die psychometrischen Eigenschaften der deutschen Version der Utrecht Work Engagement Scale 9 (UWES-9). Rehabilitation, 54, 297 303. https://doi.org/10.1055/s-0035-1555912

Schaufeli, W. B., Salanova, M., González-romá, V. \& Bakker, A. B. (2002). The measurement of engagement and burnout: A two sample confirmatory factor analytic approach. Journal of Happiness Studies, 3, 71 -92. https://doi.org/10.1023/a:1015630 930326

Schermelleh-Engel, K., Moosbrugger, H. \& Müller, H. (2003). Evaluating the fit of structural equation models: Tests of significance and descriptive goodness-of-fit measures. Methods of Psychological Research, 8 (2), $23-74$.

Schlett, C., Pauls, N. \& Soucek, R. (2018). Der Einfluss von Resilienz auf qualitative Formen der Arbeitszufriedenheit. Zeitschrift für Arbeits- und Organisationspsychologie, 62, 202-223. https://doi.org/10.1026/0932-4089/a000278

Schneider, G., Mendler, T., Heuft, G. \& Burgmer, M. (2008). Validität der Konfliktachse der Operationalisierten Psychodynamischen Diagnostik (OPD-1) - Empirische Ergebnisse und Folgerungen für die OPD-2. Zeitschrift für Psychosomatische Medizin und Psychotherapie, 54 (1), 46 - 62.

Schneider, W., Benecke, C., de la Parra, G., Freyberger, H. J. et al. (2018). Operationalisierte Psychodynamische Diagnostik. Psychotherapeut, 63, 373-380. https://doi.org/10.1007/s00278018-0305-1

Schönbrodt, F., Gollwitzer, M. \& Abele-Brehm, A. (2017). Der Umgang mit Forschungsdaten im Fach Psychologie: Konkretisierung der DFG-Leitlinien. Psychologische Rundschau, 68, 20 - 35. https://doi.org/10.1026/0033-3042/a000341
Schüßler, G. (2014). Konflikt. In W. Mertens (Hrsg.), Handbuch psychoanalytischer Grundbegriffe (4., überarb. und erw. Aufl., S. 489 - 493). Stuttgart: Kohlhammer.

Sell, C. \& Warsitz, R.-P. (2018). Dialektik der Psychotherapieforschung. Forum der Psychoanalyse, 34, 419-439. https://doi. org/10.1007/s00451-017-0286-7

Sijtsma, K. (2008). On the use, the misuse, and the very limited usefulness of Cronbach's alpha. Psychometrika, 74 (1), 107 120. https://doi.org/10.1007/s11336-008-9101-0

Spector, P., Cooper, C., Sanchez, J., O’Driscoll, M., Sparks, K., Bernin, P. et al. (2002). Locus of control and well-being at work: How generalizable are Western findings? (PSYNDEXshort). Academy of Management Journal, 45, 453-466. https://doi. org/10.5465/3069359

Statistisches Bundesamt. (2020a). Erwerbstätige. Zugriff am 14.02. 2020 unter https://www.destatis.de/DE/Themen/Arbeit/ Arbeitsmarkt/Glossar/erwerbstaetige.html

Statistisches Bundesamt. (2020b). Erwerbstätigkeit. Zugriff am 14.02. 2020 unter https://www.destatis.de/DE/Themen/Arbeit/ Arbeitsmarkt/Erwerbstaetigkeit/_inhalt.html\#sprg235976

Swider, B. W. \& Zimmerman, R. D. (2010). Born to burnout: A metaanalytic path model of personality, job burnout, and work outcomes. Journal of Vocational Behavior, 76, 487-506. https:// doi.org/10.1016/j.jvb.2010.01.003

Tangney, J. P. (1990). Assessing individual differences in proneness to shame and guilt: Development of the Self-Conscious Affect and Attribution Inventory. Journal of Personality and Social Psychology, 59 (1), 102-111. https://doi.org/10.1037/ 0022-3514.59.1.102

Thomä, H. \& Kächele, H. (2006). Psychoanalytische Therapie: Grundlagen (3., überarb. und aktual. Aufl.). Heidelberg: Springer Medizin Verlag.

Thoresen, C. J., Kaplan, S. A., Barsky, A. P., Warren, C. R. \& de Chermont, K. (2003). The affective underpinnings of job perceptions and attitudes: A meta-analytic review and integration. Psychological Bulletin, 129, 914-945. https://doi.org/10.1037/ 0033-2909.129.6.914

Turgut, S., Michel, A. \& Sonntag, K. (2014). Einflussfaktoren emotionaler Erschöpfung und Arbeitszufriedenheit. Zeitschrift für Arbeits- und Organisationspsychologie, 58, 140-154. https:// doi.org/10.1026/0932-4089/a000150

Zimmermann, J., Stasch, M., Grande, T., Schauenburg, H. \& Cierpka, M. (2014). Der Beziehungsmuster-Q-Sort (OPD-BQS): Ein Selbsteinschätzungsinstrument zur Erfassung von dysfunktionalen Beziehungsmustern auf Grundlage der Operationalisierten Psychodynamischen Diagnostik. Zeitschrift für Psychiatrie, Psychologie und Psychotherapie, 62 (1), 43 -53. https://doi.org/ 10.1024/1661-4747/a000177

\section{Historie}

Eingegangen: 15.02.2019

Revision eingegangen: 24.07.2019

Onlineveröffentlichung: 07.04.2020

\section{Danksagung}

Wir danken Johannes Bohn für die Unterstützung bei methodischen Fragen.

\section{ORCID}

Hannes Gisch

(iD)https://orcid.org/0000-0003-1515-5358 
Hannes Gisch, M. Sc.

Prof. Dr. Thomas Kretschmar

Mind Institute SE

Werderscher Markt 12

10117 Berlin

hg@dynamind.com.
Prof. Dr. Johannes Zimmermann

Institut für Psychologie

FB 01 Humanwissenschaften

Universität Kassel

Holländische Straße $36-38$

34127 Kassel 